\section{Prototyp mit Kinderkrankheiten}

Seit dem ersten Januar 2005 ist mit dem EU-Emissionshandel für Treibhausgase erstmals in der deutschen Umweltpolitik ein System handelbarer Emissionsrechte in Kraft. Nach einem Jahr lässt sich ein geteiltes Zwischenfazit ziehen, das zur Weiterentwicklung des Systems genutzt werden sollte.Von Sven Rudolph

S eit der kanadische Ökonom John Harkness Dales im Jahr 1968 ausgehend von der Kritik an der Pigou-Steuer und mit explizitem Bezug auf den eigentumsrechtlichen Ansatz von Coase die Idee handelbarer Emissionsrechte zum Schutz der natürlichen Umwelt entwickelte, erfreut sich der Handel mit Emissionszertifikaten bei Umweltökonomen aufgrund seiner ökologischen Effektivität und seiner ökonomischen Effizienz großer Beliebtheit.

\section{Späte Umsetzung in Deutschland}

Erste Erfahrungen mit einem umfassenden Einsatz des Instruments in der US-amerikanischen Luftreinhaltepolitik erwiesen sich als durchaus vielversprechend. Auch in Deutschland wurde das Instrument bereits ab der Mitte der 70er Jahre diskutiert und Anfang der 80er Jahre in die politische Debatte eingespeist. Erst mit dem EU-Emissionshandel für Treibhausgase ab dem Jahr 2005 erfolgte jedoch eine Umsetzung des Instruments in die praktische Umweltpolitik in Deutschland.

Das Grundkonzept handelbarer Emissionsrechte ist denkbar einfach: Für ein geografisches Gebiet wird eine absolute Gesamtmenge an Emissionen festgelegt. Ausgehend von dieser erhalten die betroffenen Emittenten Emissionslizenzen. Jede Emission ist nun an den Besitz eines Emissionserlaubnisscheins gebunden. Die Lizenzen sind frei übertragbar, sodass sich ein Markt für Emissionslizenzen entwickeln kann, der in der Theorie über ein einheitliches Preissignal und die Angleichung der Grenzvermeidungskosten über alle Emittenten zur volkswirtschaftlich kostenminimalen und sicheren Erreichung des ökologischen Ziel führt.

Die EU setzt den Emissionshandel für Treibhausgase, inspiriert vom US-amerikanischen Schwefeldioxid-Handel, als klassisches Cap-and-Trade-System in zwei Phasen um. Die erste reicht von 2005 bis 2007, die zweite von 2008 bis 2012. Aus dem Kyoto-Ziel der EU einer Reduktion der Treibhausgase um acht Prozent bis 2008 bzw. 2012 gegenüber 1990 werden nationale, absolute Lizenzgesamtmengen abgeleitet, die über nationale Allokationspläne (NAPs) an die betroffenen Anlagen ausgegeben werden. Die Emissionserlaubnis für eine Tonne Kohlendioxid ist nun an den Besitz einer Emissionslizenz gebunden, die wiederum frei übertragen werden kann, sodass ein europäischer Markt für Kohlendioxid entstehen kann. Dieser sorgt für eine effiziente Allokation von Emissionsrechten und Vermeidungsmaßnahmen.

\section{Ansätze zur Fortentwicklung}

Da sich der Handel in der ersten Handelsperiode auf Kohlendioxid als bedeutendstes Klimagas sowie auf die Energiewirtschaft und energieintensive Industrie als zentrale Verursacher konzentriert und zudem überwiegend die kostenlose Erstvergabe vereinbart wurde, mussten die
Ziele in den NAPs schadstoff- und sektorspezifisch konkretisiert sowie spezifische Zuteilungsregeln entwickelt werden. Während das grundlegende Handelssystem als durchaus gelungene Umsetzung der Lizenzlösungsidee gewertet werden kann, geben die Allokationspläne Anlass zur Kritik.

Umweltengagierte Akteure beklagen insbesondere die fehlende ökologische Integrität des EU-Emissionshandels, denn noch immer sind elf von 15 EULändern dramatisch weit von ihren Kyoto-Verpflichtungen entfernt. Über die Kyoto-Vereinbarung hinausgehende nationale Minderungsziele, wie etwa die Kohlendioxid-Reduktion aus dem deutschen Klimaschutzprogramm 2000, wurden in den NAPs nicht umgesetzt. Häufig erfolgte für die erste Handelsperiode 20052007 eine Orientierung der Zuteilungsmenge am Bedarf der betroffenen Industrien statt am notwendigen Klimaschutz. Dies hat zur Folge, dass Reduktionen von Treibhausgasen bis 2007 allenfalls gegenüber dem „business as usual“ zu erwarten sind. Im Vergleich zum Basisjahr 1990 bedeuten die NAPs hingegen häufig sogar Emissionszuwächse. Dies impliziert für die zweite Handelsperiode eine erhebliche Verschärfung der Minderungsanforderungen.

Vonseiten deutscher Unternehmen wird insbesondere Kritik am überbürokratischen System geäußert. Das Geflecht aus grundlegenden Zuteilungsregeln und Sonderregeln in Verbindung mit unterschiedlichen Zuteilungsmethoden vom Grandfathering auf der Basis historischer Emissionen bis hin $\mathrm{zu}$ verschiedenen Benchmark-Ansätzen ist hochkomplex. Dies verringert über Wettbewerbsbeeinträchtigungen und hohe Transaktionskosten die Effizienzvorteile des Instruments. Konkret lassen sich folgende Ansatzpunkte zur Vereinfachung sowie zur Erhöhung der ökologischen Effektivität und der ökonomischen Effizienz des Emissionshandels sowie des NAP identifizieren.

Zunächst ist angesichts der völkerrechtlich verbindlichen Kyoto-Verpflichtungen und der langfristigen ökologischen Notwendigkeit einer Reduktion der Treibhausgase um 80 Prozent bis 2050 
ein Einschwenken auf einen anspruchsvollen Emissionsminderungspfad notwendig. Dies könnte innerhalb des NAP II für Deutschland einerseits durch eine Orientierung der Gesamtmengenfestlegung für das Emissionshandelssegment an der Klimavorsorgevereinbarung der deutschen Wirtschaft aus dem Jahr 2000 erfolgen. Dort wurde eine Reduktion der Kohlendioxidemissionen um 45 Millionen Tonnen bis 2010 zugesagt. Andererseits müssen zur Erreichung der Minderungsziele in den Sektoren Haushalte und Verkehr glaubwürdige Maßnahmenbündel festgelegt werden.

\section{Versteigerungen als Chance}

Die EU-Richtlinie erlaubt für die zweite Handelsperiode die Versteigerung von zehn Prozent der Emissionslizenzen. Diese Option sollte genutzt und in ihrem Umfang langfristig ausgebaut werden. Auktionen senden unmittelbare Preissignale und belohnen automatisch Minderungsanstrengungen. Jede andere Zuteilungsmethode ist mit erheblichen Schwierigkeiten (Wettbewerbsverzerrungen, Transparenz der Verfahren usw.) belastet. Zu klären ist allerdings, welches konkrete Auktionsverfahren genutzt wird und wohin das Aufkommen einer solchen Versteigerung fließt.

Die Optionsregel mit Ex-post-Anpassung, die es Unternehmen erlaubt, eine Zuteilung auf der Basis von Benchmarks und Produktionsprognosen statt aufgrund historischer Emissionsdaten zu erhalten, wird in der Zukunft wegen der Ablehnung der Ex-post-Anpassung durch die EUKommission in dieser Form keinen Bestand haben. Entfällt aber die Ex-post-Anpassung, so können die Produktionsprognosen, die in der ersten Handelsperiode oft phantasievoll hoch ausfielen, nicht mehr korrigiert werden. Mehrzuteilungen an bestimmte Unternehmen ziehen jedoch die Verschärfung des Erfüllungsfaktors für andere Unternehmen und damit dauerhafte Wettbewerbsverzerrungen nach sich. Derzeit ist kein sinnvolles Ersatzverfahren zur Ex-post-Kontrolle absehbar, sodass allein die Streichung der Optionsregel sinnvoll scheint.
Eine stärkere Nutzung von Benchmarks als grundlegende Zuteilungsmethode für Alt- und Neuanlagen scheint zum gegenwärtigen Zeitpunkt schwierig. Grundsätzlich stellt sich die Frage, inwiefern staatliche Institutionen überhaupt über das notwendige Wissen zur Generierung sinnvoller Benchmarks verfügen können; ist doch ein zentraler Vorteil marktwirtschaftlicher Instrumente gerade die Aktivierung des allein dezentral bei den Emittenten vorhanden Wissens über die kostengünstigsten Reduktionspotenziale.

Konkret erweisen sich zudem brennstoffspezifische Benchmarks, wie sie für Neuanlagen im deutschen NAP I derzeit Anwendung finden, aufgrund der Verringerung von Anreizen zur Nutzung des Brennstoffwechsels als Vermeidungsoption als wenig sinnvoll. Produktspezifische Benchmarks hingegen werfen eine Vielzahl von Problemen bei der Bildung homogener Produktgruppen auf, die bereits im Rahmen der Ökosteuer nicht gelöst werden konnten. Allein für wenige einheitliche Produkte wie Strom sind solche Benchmarks vorstellbar.

Weitere Ansatzpunkte zur Erhöhung der ökologischen Effektivität und der ökonomischen Effizienz sind die Verringerung der Gewährungszeiträume für den Erfüllungsfaktor Eins (Early Action, Übertragungsregel, Basiszuteilungen etc.) sowie das Verbleiben von Emissionslizenzen bei den Betreibern für den Verlauf der zweiten Handelsperiode im Falle der Anlagenstilllegung, um so Anreize zur frühzeitigen Schließung alter, emissionsintensiver Anlagen zu setzen. Zudem ist, soweit möglich, eine zügige Einbeziehung der anderen Treibhausgase und eine Ausweitung des Emissionshandels auf andere Sektoren anzustreben, um auch hier die Angleichung der Grenzvermeidungskosten über alle Gase und Emittenten möglichst weitgehend zu erreichen.

\section{Fazit}

Die Erfahrungen mit dem EU-Emissionshandel sind geteilt. Zwar ist das Konzept theoretisch reizvoll und auch die grundsätzliche Umsetzung im EUEmissionshandel ist begrüßenswert. Die
Ausgestaltung des europäischen Handelssystems im Detail weist jedoch Schwächen auf. Die verabschiedeten NAPs deuten auf eine Verfehlung der Kyoto-Ziele hin und die Zuteilungsverfahren erweisen sich aufgrund des weitgehenden Verzichts auf die effizienteste Zuteilungsmethode der Versteigerung als hochkomplex.

Notwendig sind das Einschwenken auf einen anspruchsvollen Emissionsminderungspfad und die Vereinfachung der Zuteilungsregeln. Konkrete Schritte in Deutschland können die Verschärfung der Reduktionsziele gemäß der Klimavorsorgevereinbarung, die Nutzung und der Ausbau der gegebenen Versteigerungsmöglichkeiten, die Abschaffung der Optionsregel sowie der Einsatz produktspezifischer Benchmarks für Strom und wenige andere ausgewählte Produkte in Alt- und Neuanlagen sein.

Da bereits aus terminlichen Gründen Änderungen auf EU-Ebene unwahrscheinlich sind, können Verbesserungen allein im Rahmen der NAPs durchgesetzt werden. Gerade in Deutschland ist aber der Zeitrahmen hierfür sehr eng. Angesichts der politischen Rahmenbedingungen ist mit einer Diskussion des NAP II im Zeitraum von März bis Juni 2006 zu rechnen.

\section{Literatur \\ Rudolph, S.: Handelbare Emissionslizenzen - Die po- litische Ökonomie eines umweltökonomischen In- struments in Theorie und Praxis. Marburg 2005.}

AUTOR + KONTAKT Dr. Sven Rudolph ist Wissenschaftlicher Assistent am Fachgebiet Theorie öffentlicher und privater Unternehmen der Universität Kassel und Sprecher des Arbeitskreises Wirtschaft und Finanzen des BUND e.V.

Universität Kassel, FB Wirtschaftswissenschaften, Nora-Platiel-Str. 4, 34109 Kassel. E-Mail: s.rudolph@wirtschaft.uni-kassel.de 
(c) 20I0 Authors; licensee IÖW and oekom verlag. This is an article distributed under the terms of the Creative Commons Attribution Non-Commercial No Derivates License (http://creativecommons.org/licenses/by-nc-nd/3.o/), which permits unrestricted use, distribution, and reproduction in any medium, provided the original work is properly cited. 